\title{
Singlet oxygen prediction in gold nanoparticles-assisted PDT applied to a squamous cell carcinoma in the esophagus
}

\author{
I. Salas-García, F. Fanjul-Vélez, N. Ortega-Quijano, J. L. Arce-Diego \\ Applied Optical Techniques Group, TEISA Department, University of Cantabria \\ $A v$. de los Castros S/N, 39005 Santander (Spain) \\ isalas@teisa.unican.es; fanjulf@unican.es; ortegan@unican.es; arcedj@unican.es
}

\begin{abstract}
A predictive model for PDT singlet oxygen production in esophageal squamous cell carcinomas with gold nanoparticles is proposed. Differences enhance the ${ }^{1} \mathrm{O}_{2}$-mediated oxidative damage due to the optical absorption improvement by gold nanoparticles.

OCIS codes: (170.5180) Photodynamic therapy; (170.3660) Light propagation in tissues
\end{abstract}

\section{Introduction}

Photodynamic Therapy (PDT) has become a promising treatment for esophageal diseases such as superficial squamous cell carcinoma. Its noninvasive nature and the use of non ionizing radiation make this therapeutic technique especially advantageous over aggressive conventional techniques. However, a complete dosimetry for this treatment remains unsolved due to the complexity of the induced photochemical interaction and the process dependence with the different biological media [1]. The use of nanoparticles has been proposed to improve the present treatment outcome [2].

This work examines the differences produced in the photodynamic process, particularly in the production of the cytotoxic agent in charge of the cancer cells killing, by the inclusion of gold nanoparticles in a squamous cell carcinoma located in the esophagus wall. This was achieved by means of the PDT model that we present in section 2. The model takes into account the propagation of the optical radiation distribution in the esophageal tissue with gold nanoparticles embedded and the evolution of the different molecular components involved in the photochemical reaction. The results obtained show the spatial distribution of the cytotoxic agent as the treatment evolves. These results and the conclusions appear in section 3.

\section{Model}

Radiation Transport Theory (RTT) can be used to obtain the distribution of light in a three-dimensional tissue. The model assumes that the scattering events are sufficiently numerous as to the light to be considered incoherent, in such a way that polarization or interference effects can be neglected. The basic parameter of light is the specific intensity $I(\vec{r}, \hat{s})$ defined as the light power per unit area per unit solid angle. The radiation is expected to be at point $\vec{r}$, and to follow the direction $\hat{s}$. The scattering events are treated according to the scattering phase function $p\left(\hat{s} \cdot \hat{s}^{\prime}\right)$. Optical radiation comes from direction $\hat{s}^{\prime}$ and is redirected to $\hat{s}$. The differential radiation transport equation with no sources inside the tissue and in a steady-state situation is expressed in (1).

$$
\hat{s} \cdot \bar{\nabla} I(r, \hat{s})=-\left(\mu_{a}+\mu_{s}\right) I(r, \hat{s})+\frac{\mu_{s}}{4 \pi} \int_{4 \pi} p\left(\hat{s} \cdot \hat{s}^{\prime}\right) I\left(r, \hat{s}^{\prime}\right) d \Omega^{\prime}
$$

Among the different implementations of the Monte Carlo method applied to the RTT model, the one by Wang and Jacques was used in this work [3]. This implementation of the Monte Carlo model is also multi-layered, so it is possible to define several layers of different materials with their borders perpendicular to the laser beam, which is very useful due to the fact that tissues can be usually divided in different strata. The tissue sample used in this work is divided in two layers, representing a superficial squamous cell carcinoma lying on healthy esophageal tissue. The optical parameters and dimensions of both tissue layers are required to get a proper definition of the model. Among the optical parameters required are the index of refraction $n$, the absorption coefficient $\mu_{a}$, the scattering coefficient $\mu_{s}$ and the anisotropy of scattering $g$.

The inclusion of gold nanoparticles in the tumoral tissue makes also necessary to take into account their effect on light propagation. The optical absorption and scattering properties of gold nanospheres of $80 \mathrm{~nm}$ radius were obtained from their efficiency factors of absorption $Q_{a b s}$ and scattering $Q_{s c a}$, which were calculated by means of Mie's theory for homogeneous spherical nanoparticles with radius $r$ and the complex refractive index of gold [4]. 
Equations (2) and (3) show the expressions for the nanoparticles absorption and scattering coefficients respectively taking into account an homogeneous nanoparticles density $N_{T}$ in the target tissue.

$$
\begin{aligned}
& \mu_{a b s}=\pi r^{2} Q_{a b s} N_{T} \\
& \mu_{s c a}=\pi r^{2} Q_{s c a} N_{T}
\end{aligned}
$$

The photochemical reactions involved in a Type II reaction are characterized by means of a photochemical model [5] based on the stiff differential equations system (Eq. (4) to (9)). This model takes into account the different energetic transitions of the photosensitizer and provides the temporal evolution of the molecular compounds involved. Among them, the main cytotoxic agent in charge of tumoral cells destruction is known as singlet oxygen ${ }^{1} \mathrm{O}_{2}$.

$$
\begin{gathered}
\frac{d\left[S_{0}\right]}{d t}=-v \rho \sigma_{p s a}\left[S_{0}\right]-k p b\left[{ }^{1} O_{2}\right]\left[S_{0}\right]+\frac{\eta_{10}}{\tau 1}\left[S_{1}\right]+\frac{\eta_{30}}{\tau 3}[T]+\frac{\alpha s}{\tau 3}[T]\left[{ }^{3} O_{2}\right] \\
\frac{d\left[S_{1}\right]}{d t}-\frac{1}{\tau 1}\left[S_{1}\right]+v \rho \sigma_{p s a}\left[S_{0}\right] \\
\frac{d[T]}{d t}=-\frac{\eta_{30}}{\tau 3}[T]-\frac{\alpha s}{\tau 3}[T]\left[{ }^{3} O_{2}\right]+\frac{\eta_{13}}{\tau 1}\left[S_{1}\right] \\
\frac{d\left[{ }^{3} O_{2}\right]}{d t}=-\frac{\alpha s}{\tau 3}[T]\left[{ }^{3} O_{2}\right]+\frac{\eta_{0}}{\tau 0}\left[{ }^{1} O_{2}\right]+P \\
\frac{d\left[{ }^{1} O_{2}\right]}{d t}=-k p b\left[S_{0}\right]\left[{ }^{1} O_{2}\right]-k c x[R]\left[{ }^{1} O_{2}\right]-k s c[C]\left[{ }^{1}{ }^{1} O_{2}\right]-\frac{\eta_{0}}{\tau_{0}}\left[{ }^{1} O_{2}\right]+\frac{\alpha s}{\tau 3}[T]\left[{ }^{3} O_{2}\right] \\
\frac{d[R]}{d t}=-k c x\left[{ }^{1} O_{2}\right][R]+U
\end{gathered}
$$

\section{Results and discussion}

The model presented in the previous section was applied to an esophageal tissue bearing a superficial squamous cell carcinoma of $2 \mathrm{~mm}$ depth. The singlet oxygen distribution in the target tissue was firstly obtained without the inclusion of gold nanoparticles and then considering an homogeneous distribution of gold nanoparticles. The singlet oxygen produced in both cases can be compared in Fig. 1 for different temporal instants during the therapy. As it can be observed, the maximum singlet oxygen concentration is produced at the beginning of the treatment in both situations (Figs. 1a) and 1b)) and decreases as the irradiation time increases. The singlet oxygen is almost equal in both cases as time passes, except at the end of treatment as can be checked if Figs. 1e) and $1 \mathrm{f}$ ) are compared. At this time the concentration of singlet oxygen is not only higher when incorporating nanoparticles, but also its distribution in the target tissue varies substantially. Thus the improvement in the optical absorption caused by the administration of gold nanoparticles is not reflected in the production of singlet oxygen until the photosensitizer has been substantially photobleached. The ${ }^{1} \mathrm{O}_{2}$-mediated oxidative damage in the esophageal disease we are dealing with is enhanced as a consequence of the effect of gold nanoparticles in the optical absorption. 

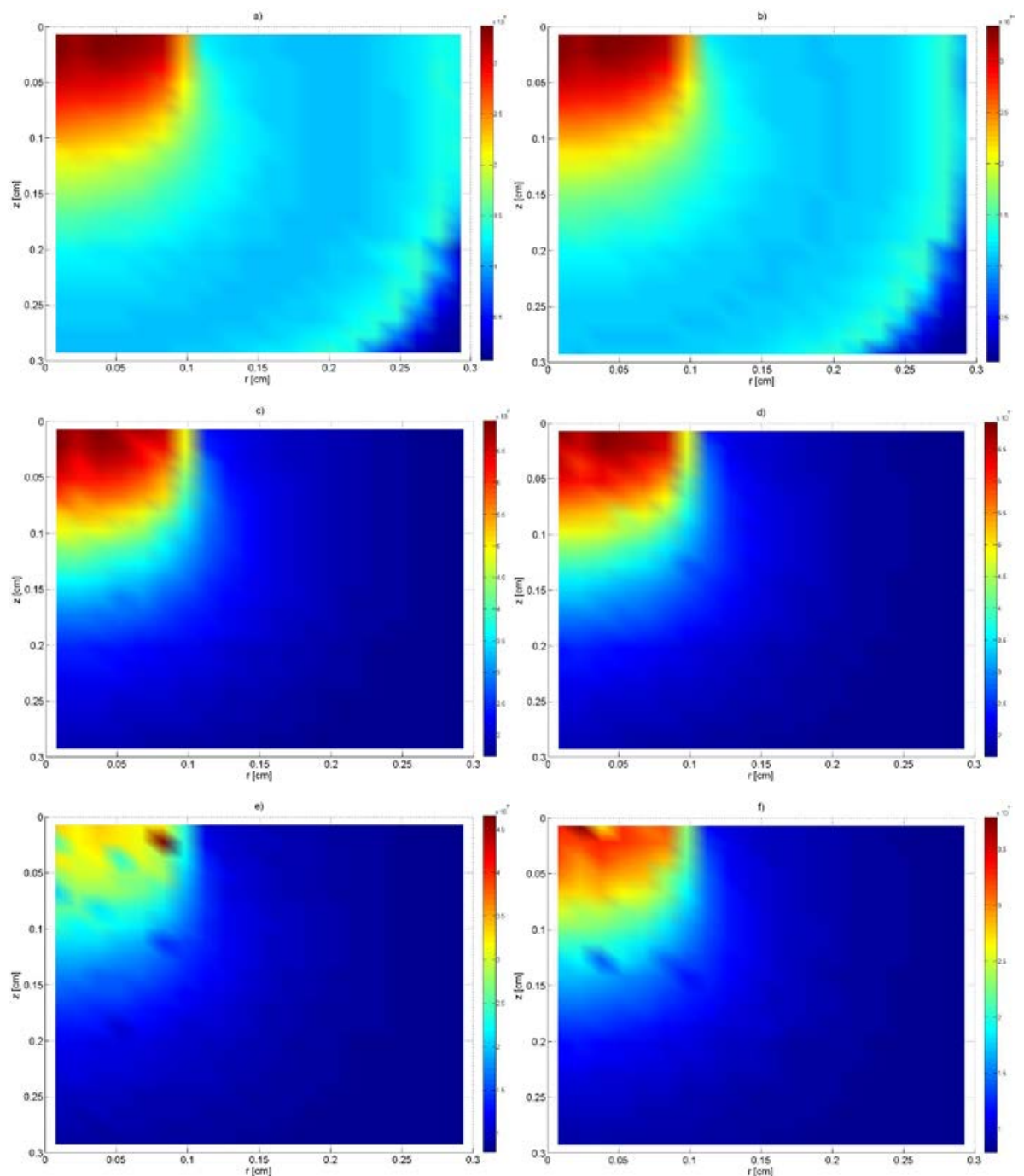

Fig.1. Singlet oxygen concentration $\left(\mathrm{cm}^{-3}\right)$ in a squamous cell carcinoma located in the esophagus wall without nanoparticles at a) $6 \mathrm{~s}$, c) $300 \mathrm{~s}$ and e) $600 \mathrm{~s}$ from the beginning of PDT and with nanoparticles at b) $6 \mathrm{~s}$, d) $300 \mathrm{~s}$ and f) $600 \mathrm{~s}$.

The PDT model proposed in this work made it possible to examine the differences provoked in the production of singlet oxygen by the inclusion of gold nanoparticles in a squamous cell carcinoma located in the esophagus wall. Results show clear differences in the cytotoxic agent distribution at the end of treatment.

\section{Acknowledgements}

This work has been partially supported by the project MAT2012-38664-C02-01 of the Spanish Ministery of Economy and Competitiveness.

\section{References}

[1] R. Bays, G. Wagnières, D. Robert, D. Braichotte, J. F. Savary, P. Monnier and H. van den Bergh, "Light Dosimetry for Photodynamic Therapy in the Esophagus” Lasers in Surgery and Medicine 20, 290-303 (1997).

[2] M. R. Hamblin and P. Mróz, Advances in Photodynamic Therapy. Basic, translational and clinical, (Engineering in medicine \& Biology, 2008).

[3] L. Wang, S. L. Jacques and L. Zheng, L., "MCML - Monte Carlo modeling of light transport in multi-layered tissues" Computer methods and programs in biomedicine 47, 131-146 (1995).

[4] C. F. Bohren and D. R. Huffman, D.R., Absorption and Scattering of Light by Small Particles (John Wiley, New York,1983).

[5] I. Salas-García, F. Fanjul-Vélez and J. L. Arce-Diego, "Photosensitizer absorption coefficient modeling and necrosis prediction during Photodynamic Therapy” Journal of Photochemistry and Photobiology B: Biology 114, 79-86 (2012). 